\title{
A Survey of Human Computation Systems
}

\author{
Man-Ching Yuen \\ Dept. of Computer Science \& Engineering \\ The Chinese University of Hong Kong \\ connieymc@gmail.com
}

\author{
Ling-Jyh Chen \\ Institute of Information Science \\ Academia Sinica \\ cclljj@iis.sinica.edu.tw
}

\author{
Irwin King \\ Dept. of Computer Science \& Engineering \\ The Chinese University of Hong Kong \\ king@cse.cuhk.edu.hk
}

\begin{abstract}
Human computation is a technique that makes use of human abilities for computation to solve problems. The human computation problems are the problems those computers are not good at solving but are trivial for humans. In this paper, we give a survey of various human computation systems which are categorized into initiatory human computation, distributed human computation and social game-based human computation with volunteers, paid engineers and online players. For the existing large number of social games, some previous works defined various types of social games, but the recent developed social games cannot be categorized based on the previous works. In this paper, we define the categories and the characteristics of social games which are suitable for all existing ones. Besides, we present a survey on the performance aspects of human computation system. This paper gives a better understanding on human computation system.
\end{abstract}

\section{INTRODUCTION}

Human computation is an idea of solving the difficult artificial intelligence (AI) problems through human power. There exists some AI problems that computers are either unable to or are very poor at solving, but they are easy for human to solve. The information collected by human computation systems is useful for machine learning systems. The existing human computation systems can be categorized as follows:

1) Initiatory Human Computation - Early, the human computation systems were proposed to collect commonsense knowledge through the use of computational power of computers and their users.

2) Distributed Human Compuation - In order to collect more information, distributed human computation systems were proposed later. It encouraged the huge number of Internet users to contribute information to the systems. However, they have no mechanism to guarantee that the information collected is accurate, and these systems are difficult to scale up.

3) Social Game-based Human Computation - According to a report published at 2006 by International Game Design Association (IGDA), an estimated 200 million people play casual games online every month [3]. To encourage more Internet users to provide accurate information to solve the difficult AI problems, social game were proposed to provide entertainment to the online game players, but as a side effect of their playing, accurate information can be collected from the players for solving AI problems. The early developed social games either rely on contributions from online volunteers or pay for the engineers to enter information. To increase the scalability of the system, social games developed later rely on the Internet online players.

The rest of this paper is organized as follows. Section II introduces the previous works on initiatory human computation system. Section III gives a brief survey on distributed human computation system. Section IV presents a survey on social game-based human computation systems which rely on volunteers or paid engineers. Section V presents the study on social games for online players. It describes the categories and the characteristics of social games. It presents how the existing games be categorized based on our categorization. Section VI presents the survey on the performance aspects on evaluating the human computation systems. Section VII gives a discussion and conclusion of our work.

\section{INITIATORY HUMAN COMPUTATION}

As computation power increases rapidly recently, computers often outperform people on tasks that are tedious, boring and time-consuming. However, some tasks that are natural for humans can be extremely difficult for computers. These tasks are usually information collection, and these information are very useful for Artificial Intelligence system or Machine Learning system. Some works are proposed for human computation.

CAPTCHA [46] is a computer generated challenge-response test to distinguish humans from computers using a common sense problem. reCAPTCHA [7] [53] is a novel CAPTCHA that produces valuable common sense knowledge to improve the OCR quality in digitizing books for the Internet Archive [4]. It works by combining two words that OCR cannot read into a challenge. One word is already identified and serves as a conventional CAPTCHA, while the other word is not identified yet. If a user recognizes the identified word, the answer to the unidentified word is assumed to be correct, and is collected to identify the unidentified word. The OCR result is therefore improved. Using games or CAPTCHA to collect human common sense knowledge, as described above, are innovative ideas. They are very suitable for large scale applications such as labeling images for a portal of useruploaded images and the Internet Archive project [4].

KA-CAPTCHA [14] is an extension of the popular CAPTCHA method. Its contribution is based on the observation that every correct answer submitted by humans to the CAPTCHA test in indeed a solution to a problem that computers are unable to solve. Therefore, CAPTCHA solvers 
are highly interested in providing a valid response to the CAPTCHA test (because they want to access the protected resource), then an efficient knowledge acquisition mechanism can be employed by posing not a random question to the test taker, but by strategically asking for a solution to a particular open problem that is of interest to the CAPTCHA designer.

\section{Distributed Human COMPUTATION}

To encourage hundreds of millions of Internet users to contribute to solve the difficult AI problems, some distributed human computation works were proposed in previous works.

- Some anti-spam mechanisms such as Vipul's Razor $^{1}$ use human votes to determine if a given email is spam.

- The distributed proof-readers project Proofreader $^{2}$ is geared towards eliminating optical character recognition errors in Project Gutenberg ${ }^{3}$ electronic books. The idea is to give a (small) portion of the image file and corresponding text (generated by OCR) side-by-side to a human proofreader. The proofreader in turn provides edited text to fix any errors. By giving the same piece of text to several proofreaders, errors can be reliably eliminated.

- Wikipedia ${ }^{4}$ are online encyclopedias that are written by Internet users, and the writing is distributed in that essentially almost anyone can contribute to the Wiki.

- Yahoo! Answers ${ }^{5}$ is a general question-answering forum to provide automated collection of human reviewed data at Internet-scale. These human-reviewed data are often required by enterprise and web data processing.

- Yahoo! Suggestion Board ${ }^{6}$ is an Internet-scale feedback and suggestion system.

- Amazon Mechanical Turk [1] provides monetary rewards (at least one cent) for tasks, can be used for generic task, and there is no explicit collaboration between answerers.

- LabelMe [35] is a web-based tool for image annotation. Anybody can annotate data using this tool and thus contribute to constructing a large database of annotated objects. The incentive to annotate data is the data itself. You can only have access to the database once you have annotated a certain number of images.

- The website 43Things ${ }^{7}$ also collects goals from users, and in turn it provides a way for users to find other users who have the same goals, even if they are uncommon.

- Yahoo's flickr ${ }^{8}$ is a popular photo-sharing site and provides a mechanism for users to caption their photos. These captions are already being used as alternative text.

- There are two recently developed music annotation games, MajorMiner [28] and The Listen Game [43]. MajorMiner is playing offline against a database, while The Listen Game simultaneously against multiple players.

\footnotetext{
${ }^{1}$ Vipuls razor web site, http://sourceforge.net/projects/razor

${ }^{2}$ The distributed proofreaders project, http://www.pgdp.net/c/

${ }^{3}$ Project gutenberg, http://www.gutenberg.net/

${ }^{4}$ The free encyclopedia, http://en.wikipedia.org

${ }^{5}$ Yahoo! answers, http://answers.yahoo.com/

${ }^{6}$ Yahoo! Suggestion Board, http://suggestions.yahoo.com/

${ }^{7} 43$ things website for collecting goals from users, http://www.43things.com/

${ }^{8}$ Yahoos flickr, http://www.flickr.com/
}

\section{Social Game-based Human Compuation with VOLUNTEERS OR PAID ENGINEERS}

Early, there were a number of projects tried to solve many difficult AI problems through the use of computational power of computers and their users around the world.

Cyc [24] aims to collect information from the input by paid knowledge engineers. Its disadvantage is to pay for the knowledge engineers to input information while there is no guarantee that the information they input is correct. Open Mind [6] [41] is a world-wide collaborative effort initiated by the MIT Media Lab to collect common sense knowledge from people to develop intelligent software. The information gathered from regular Internet users are fed to machine learning algorithms. Open Mind costs very little. However, it is too reliance on the willingness of unpaid volunteers to donate their time to contribute information, thus it is unable to scale up. Besides, it cannot guarantee that the information they enter is accurate. Mindpixel [5] relies on ordinary Internet users. It has a collaborative system in which many participants create and classify a statement as true or false, thus building up a large database of facts. It rewards those who consistently validate a fact inline with the other users. Thus, it can guarantee that the information they enter is accurate but its costs is very high.

Wildfire wally [33] is a volunteer computing causal game capable of solving the maximum clique problem. However, again, it is too reliance on the willingness of unpaid volunteers to donate their time to contribute information.

\section{Social Game-based Human Compuation with ONLINE PLAYERS}

The concept of "Human Computation Game" or "Social Game" was pioneered by Luis Von Ahn and his colleagues, who created games with a purpose [44], which people play voluntarily. The games produce useful metadata as a by-product. By taking advantage of people's desire to be entertained, human computation solve some problems that computer computation cannot currently resolve completely.

\section{A. Game Properties}

The current social games are categorized by game structure, verification method, game mechanism and player requirement. Table I shows the categorization of social games. In the following, we describe the characteristic of each category.

1) Game Structure: Game structure defines the key elements of a game including the input of players, the output of players, the relationship among the input and output of all players, and the winning condition. In [48], von Ahn defined three types of games, and they are output-agreement game, input-agreement game and inversion-problem game. We find out that some recent social games cannot be categorized into any one of the three categories and these games aim for collecting players' output pattern or behavior. We define a new category, output-optimization game, for these games.

In Output-agreement Game, all players are given the same input and must produce outputs based on the common input. In Input-agreement Game, all players are given inputs that 
TABLE I

CATEGORIZATION OF SOCIAL GAMES

\begin{tabular}{|c|c|c|}
\hline Game Structure & Verification Method & Game Mechanism \\
\hline \hline Output-agreement & Symmetric & Collaborative or Hybrid \\
\hline Input-agreement & Symmetric & Collaborative or Hybrid \\
\hline Inversion-problem & Asymmetric & Collaborative or Competitive or Hybrid \\
\hline Output-optimization & Symmetric or Asymmetric & Collaborative or Competitive or Hybrid \\
\hline
\end{tabular}

are known by the game (but not by the players) to be the same or different. The players are instructed to produce outputs describing their input, so their partners are able to assess whether their inputs are the same or different. Players see only each other's outputs. In Inversion-problem Game, the first player has access to the whole problem and gives hints to the second player to make a guess. If the second player is able to guess the secret, we assume that the hints given by the first player are correct. In Output-optimization Game, all players are given the same input and their outputs are the hints of other players' outputs.

2) Verification Method: It defines the method to check the output accuracy of players by asking players to do the same tasks or different tasks in a game.

In Symmetric Verification Game, all players are asked to do the same thing and their outputs are checked against each other. In Asymmetric Verification Game, all players are asked to do different tasks and their outputs are checked against each other.

3) Game Mechanism: It defines the relationship of all players in the game in order to achieve the winning condition.

In Collaborative Game, to achieve the winning condition of all players, a player has to complete his assigned task which is helping other players to complete their tasks. In Competitive Game, to achieve the winning condition of a player, a player has to complete his assigned task. His achievement is compared with other players' achievement or his history of game records or information stored in a database. In Hybrid Game, to achieve the winning condition of some players, players have to complete their assigned tasks which are helping the others to complete their tasks. Then, the achievements of all players are compared among each other or their history of game records or information stored in a database.

4) Player Requirement: Player requirement defines the rules on accessing the game of all players: (1) players accessing the game at different time period are allowed or not; (2) the number of players is required in a game.

In Synchronous Game, players access the game at the same time. Players have to give real-time response to other players' action. In Asynchronous Game, players do not have to give real-time response to other players' action. The information collected from one player is stored in a database and will be used to determine the correctness of other players' output.

Single-player Game allows one player plays in a game. Moves of the other can be simulated from the prerecorded game. Two-player Game allows two players play in a game. Multi-player Game allows multiple players play in a game.

\section{B. Type One: Output-agreement games}

All players are given the same input and must produce outputs based on the common input. The verification method used by output-agreement games is symmetric because all players are asked to do the same things and their outputs are checked against each other. Because of using symmetric verification, the game mechanism for a two-player output-agreement must be collaborative; while for a multi-player output-agreement, it can be either collaborative or hybrid. Besides, ouputagreement game can be synchronous or asynchronous game. For instance, Gopher Game [12] is an asynchronous multiplayer game, because it is not necessary for all players to be connected to the system at the same time and the outputs of all players are stored in the database of the system for verification.

\section{Type Two: Input-agreement games}

All players are given inputs that are known by the game (but not by the players) to be the same or different. The players are instructed to produce outputs describing their input, so their partners are able to assess whether their inputs are the same or different. Players see only each other's outputs. The verification method used by input-agreement games is symmetric because all players are asked to do the same things in the game. Because of using symmetric verification, the game mechanism for a two-player input-agreement must be collaborative; while for a multi-player input-agreement, it can be either collaborative or hybrid. TagATune [23] is a typical example of input-agreement game.

\section{Type Three: Inversion-problem games}

The first player has access to the whole problem and gives hints to the second player to make a guess. If the second player is able to guess the secret, we assume that the hints given by the first player are correct. Since all players are asked to do different tasks and their outputs are checked against each other, inversion-problem game uses asymmetric verification method. This type of game can be collaborative, competitive or hybrid.

\section{E. Type Four: Output-optimization games}

All players are given the same input and their outputs are the hints of other players' outputs. This type of game can use either symmetric or asymmetric verification method. When symmetric verification is used, it must be a collaborative game, for instance, Restaurant Game [32]. When asymmetric verification is used, it can be a competitive or hybrid game, for instance, Diplomacy [21]. 
TABLE II

EXAMPLES OF SOCIAL GAMES

\begin{tabular}{|c|c|c|c|c|c|}
\hline \multirow{2}{*}{ Game Structure } & \multirow{2}{*}{ Verification Method } & \multirow{2}{*}{ Game Mechanism } & \multicolumn{2}{|c|}{$\begin{array}{l}\text { Player Requirement } \\
\end{array}$} & \multirow{2}{*}{ Examples } \\
\hline & & & Num of Player & Game Play & \\
\hline \multirow{3}{*}{ Output-agreement } & \multirow{3}{*}{ Symmetric } & Collaborative & 2 & Synchronous & ESP, Matchi, Squigl, OntoGame \\
\hline & & Hybrid & Multi-players & Synchronous & Common Consensus, Social Heroes \\
\hline & & Hybrid & Multi-players & Asynchronous & Gopher Game \\
\hline \multirow{2}{*}{ Input-agreement } & \multirow{2}{*}{ Symmetric } & Collaborative & 2 & Synchronous & TagATune \\
\hline & & Hybrid & N/A & N/A & N/A \\
\hline \multirow{3}{*}{ Inversion-problem } & \multirow{3}{*}{ Asymmetric } & Collaborative & 1 or 2 & Synchronous & Peekaboom, Verbosity \\
\hline & & Competitive & 2 & Asynchronous & Dogear, CyPRESS, CARS \\
\hline & & Hybrid & 1 or Multi-players & Synchronous & Phetch \\
\hline \multirow{6}{*}{ Output-optimization } & \multirow{3}{*}{ Symmetric } & Collaborative & 2 & Synchronous & Restaurant Game \\
\hline & & Competitive & $\mathrm{N} / \mathrm{A}$ & N/A & N/A \\
\hline & & Hybrid & Multi-players & Synchronous & Diplomacy \\
\hline & \multirow{3}{*}{ Asymmetric } & Collaborative & N/A & N/A & N/A \\
\hline & & Competitive & N/A & N/A & N/A \\
\hline & & Hybrid & N/A & N/A & N/A \\
\hline
\end{tabular}

\section{F. Existing social games}

Table II presents examples of social games based on the categorization.

The online ESP Game [47] was the first human computation system, and it was subsequently adopted as the Google Image Labeler ${ }^{9}$. Its objective is to collect labels for images on Web.

In addition to image annotation, the Peekaboom system [52] can help determine the location of objects in images; and the Squigl system [2] provides complete outlines of the objects in an image. Phetch [49], [50] provides image descriptions that improve web accessibility and image searches, while the Matchin system [2] helps image search engines rank images based on which ones are the most appealing. The concept of the ESP Game has been applied to other problems. For instance, the TagATune system [23], which provides annotation for sounds and music, can improve audio searches. The Verbosity system [51] and the Common Consensus system [25] collect "common-sense" knowledge that is valuable for commonsense reasoning and enhancing the design of interactive user interfaces.

There currently exist many social bookmark sites on the Internet, such as del.icio.us ${ }^{10}$ [17]. Dogear Game [15] is designed to accomplish organization goals. Individual players receive entertainment and learn about their colleagues' bookmarks. It uses the bookmarks in an enterprise socialbookmarking system called Dogear [31].

Several GWAP-based geospatial tagging systems have been proposed in recent years. MobiMission [18] is a location-based pervasive social game in which missions are created, solved, and reviewed by players. The system does its best to assign "nearby" missions to players (e.g., find a good cafe near the Empire State Building). If there are no nearby missions, it assigns "location-independent" missions instead (e.g., take a picture of a tall building). Similarly, in the Gopher game [12], missions are created, solved, and reviewed by players, except that the system assigns missions to "nearby" players

\footnotetext{
${ }^{9}$ Google Image Labeler, http://images.google.com/imagelabeler/

${ }^{10} \mathrm{http} / / /$ del.icio.us/
}

only. CityExplorer [29], [30] is a location-based variant of the popular board game Carcassonne ${ }^{11}$. It only allows players to place tokens (followers) in predefined types of real world locations, so players are "forced" to explore the unstructured game area, and the system can collect specific geospatial data as a by-product. Moreover, Eyespy [8] allows players to tag geographic locations with photos or text; or 'confirm' the locations of places that other players have tagged. As a result, Eyespy produces a collection of recognizable and locatable geographic details for location-based applications.

The Context-Aware Recognition Survey (CARS) system [55] uses ubiquitous sensors to monitor activities in the home. Moreover, [39] applies human computation to ontology alignment and web content annotation for the Semantic Web using various games, such as OntoPronto, SpotTheLinks, OntoTube.

CyPRESS [22] is an online game used for e-recruiting to process job applications. It combines the two approaches of self- and e-assessment. In the first step, potential applicants should be encouraged to assess their own appropriateness in regard to the job vacancy (self-assessment). In the second step, it enables an efficient pre-selection of the applicants via company-specific tasks in an e-assessment-center.

Restaurant Game [32] presents a method of learning human behavior patterns through online gaming. In the game, players collaborate to create a salad through selecting and discussing available salad items, and the collected data is intended for learning behavior models for autonomous social robots. Diplomacy [21] is a strategic board game with strong emphasis on cooperation and strategizing with opponents for ultimate victory. Players are required to make deals and plan together with their opponents by creating and dissolving alliances from round to round. Social Heroes [38] is a pervasive social game in which players trade points by tagging each other using Twitter. Social Heroes provides an interface for surrounding personal relationships, identity and communication.

Besides the characteristics of human computation games, recently there are some human computation approaches other

\footnotetext{
${ }^{11}$ Carcassonne (German only), http://www.carcassonne.de/
} 
than games are proposed, which are shown as follows.

Since converting an AI problem into a game is nontrivial [45], the first general human computation framework was proposed in [56]. By adopting a Web 2.0 approach, the framework binds its human computation system, problems providers, participating Web sites and Internet users together to label images and video efficiently but it is not for solving other large-scale human computation problems. The concept of secure distributed human computation was studied [16]. It used basic probability tools to analyze how many malicious parties such a system can tolerate. In [27], it presented the Shared Virtual Environment (SVE), the framework to be used for the development of two types of applications (social gaming and collaborative work) where augmentation was studied.

Human computation games require conscious attention and explicit intent to perform the specific task. However, there is a set of tasks that can be usefully performed by humans even when they are not explicitly trying to perform them. Shenoy and Tan [37] proposed an approach using an electroencephalograph (EEG) device to measure the presence and outcomes of implicit cognitive processing, processing that users perform automatically and may not even be aware of, to perform image classification. It showed that the classification accuracies are very high However, the costs of EEG devices are high.

To help visually impaired people surf web pages, WebInSight [10] is a system that automatically creates and inserts alternative text into web pages on-the-fly. It caches alterative text in a local database and can add new labels seamlessly after a web page is downloaded, resulting in minimal impact to the browsing experience. In [11], it aims to provide the characters with social skills, including the ability to interact using natural language. In a social-personal information management (SPIM) system [34], it explored the idea of tagging people for selective information sharing. People tagged with the same key word form a relationship group that can be used as an access control option for each piece of information.

\section{The Performance Aspect of Human COMPUTUTAION}

In addition to designing new human computation systems, several studies have investigated the performance aspect of human computation recently [9], [13], [16], [20], [26], [36], [40], [42], [48], [54]. For example, in [16], Gentry et al. analyzed the security and reliability against malicious parties on distributed human computation systems, and derived a set of design principles using standard decision-theory concepts. On the other hand, $\mathrm{Su}$ et al. [42] performed comprehensive experiments using real datasets to study the impacts of user behavior on the quality of human-reviewed data, and they concluded that the quality of the collected data improves significantly if worker prequalification mechanisms are applied.

Using Amazon Mechanical Turk [1] as an example, [40] compared the quality of non-expert annotations and existing gold standard labels provided by expert labelers. The results demonstrated that it is required to collect an average of 4 non-expert labels per item in order to emulate expert-level label quality, and that the annotation quality can be improved significantly after applying bias correction techniques. In addition, Sheng et al. [36] proposed an analysis to model the data quality using repeated labeling with a cost. They found that, with repeated labeling, it is possible to improve the data quality at low cost, especially when labels are noisy. Moreover, when the cost of processing the unlabeled data is not free, repeated labeling is preferable in that it is effective and robust in providing labels of good quality.

In [54], Weber et al. presented a machine learning-based model that can play with the ESP game without looking at the image. Based on the model, they proposed an enhanced scoring system for the ESP game to encourage users to contribute less predicable labels and therefore improve the quality of the collected labels. [19] and [20] presented gametheoretic analysis for the ESP game and the PhotoSlap game respectively, and they investigated the equilibrium behavior under different incentive mechanisms. [48] proposed a set of evaluation metrics, such as throughput, lifetime play, and expected contribution, to determine the effectiveness of ESP-like GWAP systems. Meanwhile, [13] proposed another metric, called system gain, to measure the performance of ESP-like GWAP systems, and they designed an optimal puzzle selection algorithm to improve the ESP game in terms of system gain. [26] proposed an analytical model for generalized ESP game, where the number of players, the consensus threshold, and the stopping condition are all variable. It argued that, by applying this model, ESP game can be further extended and optimized.

\section{CONCLUSION}

We have surveyed various human computation systems, and categorized them into four types: initiatory human computation, distributed human computation and social game-based human computation with volunteers, paid engineers and online players. Moreover, we compare the emerging social-gamebased human computation systems based on the game structure, verification method, and game mechanism; and present the performance aspect issues of human computation systems. To the best of our knowledge, this is the first extensive survey of the emerging human computation issue. This survey not only provides a better understanding about human computation systems, but also facilitates future research activities and application developments in the field of human computation.

\section{ACKNOWLEDGMENT}

The work described in this paper is supported by grants from Microsoft (Project No.: 6902498). This work is also affiliated with the Microsoft-CUHK Joint Laboratory for Human-centric Computing and Interface Technologies.

\section{REFERENCES}

[1] Amazon mechanical turk. https://www.mturk.com/.

[2] GWAP. http://www.gwap.com/gwap/.

[3] International game developers association, 2006 casual games white paper. http://www.igda.org/.

[4] Internet archive. http://www.archive.org/index.php.

[5] Mindpixel project. http://www.mindpixel.org.

[6] The open mind project. http://www.openmind.org. 
[7] recaptcha. http://recaptcha.net/.

[8] M. Bell, S. Reeves, B. Brown, S. Sherwood, D. MacMillan, J. Ferguson, and M. Chalmers. Eyespy: Supporting navigation through play. In $A C M$ SIGCHI, 2009.

[9] P. N. Bennett, D. Maxwell, and A. Mityagin. Learning Consensus Opinion: Mining Data from a Labeling Game. In WWW, 2009.

[10] J. P. Bigham, R. S. Kaminsky, R. E. Ladner, O. M. Danielsson, and G. L. Hempton. WebInSight: making web images accessible. In ACM SIGACCESS Conference on Assistive Technologies, pages 181$188,2006$.

[11] J. Brusk. Dialogue management for social game characters using statecharts. In M. Inakage and A. D. Cheok, editors, Advances in Computer Entertainment Technology, volume 352 of ACM International Conference Proceeding Series, pages 219-222. ACM, 2008.

[12] S. Casey, B. Kirman, and D. Rowland. The gopher game: a social, mobile, locative game with user generated content and peer review. In International Conference on Advances in Computer Entertainment Technology, pages 9-16, 2007.

[13] L.-J. Chen, B.-C. Wang, K.-T. Chen, I. King, and J. H.-M. Lee. An analytical study of puzzle selection strategies for the esp game. In IEEE/WIC/ACM Web Intelligence Conference, 2008.

[14] B. N. da Silva and A. C. B. Garcia. Ka-captcha: An opportunity for knowledge acquisition on the web. In $A A A I$, pages 1322-1327. AAAI Press, 2007.

[15] C. Dugan, M. J. Muller, D. R. Millen, W. Geyer, B. Brownholtz, and M. Moore. The dogear game: a social bookmark recommender system. In T. Gross and K. Inkpen, editors, GROUP, pages 387-390. ACM, 2007.

[16] C. Gentry, Z. Ramzan, and S. G. Stubblebine. Secure distributed human computation. In ACM Conference on Electronic Commerce, pages 155164, 2005.

[17] S. A. Golder and B. A. Huberman. The structure of collaborative tagging systems. CoRR, abs/cs/0508082, 2005.

[18] L. Grant, H. Daanen, S. Benford, A. Hampshire, A. Drozd, and C. Greenhalgh. MobiMissions: the game of missions for mobile phones. In ACM SIGGRAPH, 2007.

[19] C.-J. Ho, T.-H. Chang, and J. Y. jen Hsu. PhotoSlap: A Multi-player Online Game for Semantic Annotation. In AAAI, 2007.

[20] S. Jain and D. C. Parkes. A Game-Theoretic Analysis of Games with a Purpose. In Workshop on Internet and Network Economics, 2008.

[21] A. Krzywinski, W. Chen, and A. Helgesen. Agent architecture in social games the implementation of subsumption architecture in diplomacy. In C. Darken and M. Mateas, editors, AIIDE. The AAAI Press, 2008.

[22] S. Laumer, A. von Stetten, A. Eckhardt, and T. Weitzel. Online gaming to apply for jobs - the impact of self- and e-assessment on staff recruitment. In HICSS, pages 1-10. IEEE Computer Society, 2009.

[23] E. Law and L. von Ahn. Input-agreement: A new mechanism for data collection using human computation games. In ACM CHI, 2009.

[24] D. B. Lenat. Cyc: a large-scale investment in knowledge infrastructure. Commun. ACM, 38(11):33-38, 1995.

[25] H. Lieberman, D. Smith, and A. Teeters. Common Consensus: a webbased game for collecting commonsense goals. In ACM Workshop on Common Sense for Intelligent Interfaces, 2007.

[26] C.-W. Lin, K.-T. Chen, L.-J. Chen, I. King, and J. H.-M. Lee. An Analytical Approach to Optimizing The Utility of ESP Games. In IEEE/WIC/ACM International Conference on Web Intelligence, 2008.

[27] M. C. J. Lizandra, M. A. Raya, L. Gamberini, I. Zaragozá, and F. Martino. Shared virtual environment (sve): a framework for developing social games. In M. Inakage, N. Lee, M. Tscheligi, R. Bernhaupt, and S. Natkin, editors, Advances in Computer Entertainment Technology, volume 203 of ACM International Conference Proceeding Series, pages 258-259. ACM, 2007.

[28] M. Mandel and D. Ellis. A web-based game for collecting music metadata. In 8th International Conference on Music Information Retrieval (ISMIR).

[29] S. Matyas. Playful geospatial data acquisition by location-based gaming communities. The International Journal of Virtual Reality, 6(3):1-10, 2007.

[30] S. Matyas, C. Matyas, C. Schlieder, P. Kiefer, H. Mitarai, and M. Kamata. Designing Location-based Mobile Games With A Purpose: Collecting Geospatial Data with CityExplorer. In ACM ACE, 2008.

[31] D. R. Millen, J. Feinberg, and B. Kerr. Dogear: Social bookmarking in the enterprise. In R. E. Grinter, T. Rodden, P. M. Aoki, E. Cutrell, R. Jeffries, and G. M. Olson, editors, CHI, pages 111-120. ACM, 2006.
[32] J. Orkin and D. Roy. The restaurant game: Learning social behavior and language from thousands of players online. Journal of Game Development, 3(1):39-60, December 2007.

[33] E. Peck, M. Riolo, and C. Cusack. Wildfire wally: a volunteer computing game. In Future Play '07: Proceedings of the 2007 conference on Future Play, pages 241-242, New York, NY, USA, 2007. ACM.

[34] M. N. Razavi and L. Iverson. Supporting selective information sharing with people-tagging. In M. Czerwinski, A. M. Lund, and D. S. Tan, editors, CHI Extended Abstracts, pages 3423-3428. ACM, 2008.

[35] B. C. Russell, A. Torralba, K. P. Murphy, and W. T. Freeman. LabelMe: A Database and Web-Based Tool for Image Annotation. International Journal of Computer Vision, 77(1-3):157-173, May 2008.

[36] V. S. Sheng, F. Provost, and P. G. Ipeirotis. Get Another Label? Improving Data Quality and Data Mining Using Multiple, Noisy Labelers. In $A C M K D D, 2008$.

[37] P. Shenoy and D. S. Tan. Human-aided computing: utilizing implicit human processing to classify images. In ACM SIGCHI Conference on Human Factors in Computing Systems, 2008.

[38] A. Simon. Social heroes: games as apis for social interaction. In S. Tsekeridou, A. D. Cheok, K. Giannakis, and J. Karigiannis, editors, DIMEA, volume 349 of ACM International Conference Proceeding Series, pages 40-45. ACM, 2008.

[39] K. Siorpaes and M. Hepp. Games with a Purpose for the Semantic Web. IEEE Intelligent Systems, 23(3):50-60, May/June 2008.

[40] R. Snow, B. O’Connor, D. Jurafsky, and A. Y. Ng. Cheap and Fast - But is it Good? Evaluating Non-Expert Annotations for Natural Language Tasks. In International Conference on Empirical Methods in Natural Language Processing, 2008.

[41] D. Stork and L. C.P. Open mind animals: Ensuring the quality of data openly contributed over the world wide web. In Learning from Imbalanced Data Sets: Papers from the AAAI Workshop, pages 4-9, 2000.

[42] Q. Su, D. Pavlov, J.-H. Chow, and W. C. Baker. Internet-scale collection of human-reviewed data. In International World Wide Web Conference, pages 231-240, 2007

[43] D. Turnbull, R. Liu, L. Barrington, and G. Lanckriet. A gamebased approach for collecting semantic annotations of music. In 8th International Conference on Music Information Retrieval (ISMIR).

[44] L. von Ahn. Games with a Purpose. IEEE Computer, 39(6):92-94, June 2006.

[45] L. von Ahn. Human computation. PhD Thesis, December 2005.

[46] L. von Ahn, M. Blum, N. J. Hopper, and J. Langford. Captcha: Using hard ai problems for security. In E. Biham, editor, EUROCRYPT, volume 2656 of Lecture Notes in Computer Science. Springer, 2003.

[47] L. von Ahn and L. Dabbish. Labeling Images with a Computer Game. In ACM SIGCHI Conference on Human Factors in Computing Systems, 2004.

[48] L. von Ahn and L. Dabbish. Designing games with a purpose. Communications of the ACM, 51(8):58-67, August 2008.

[49] L. von Ahn, S. Ginosar, M. Kedia, and M. Blum. Improving Image Search with PHETCH. In IEEE International Conference on Acoustics, Speech and Signal Processing, 2007.

[50] L. von Ahn, S. Ginosar, M. Kedia, R. Liu, and M. Blum. Improving accessibility of the web with a computer game. In ACM SIGCHI Conference on Human Factors in Computing Systems, 2006.

[51] L. von Ahn, M. Kedia, and M. Blum. Verbosity: A Game for Collecting Common-Sense Facts. In ACM SIGCHI Conference on Human Factors in Computing Systems, 2006.

[52] L. von Ahn, R. Liu, and M. Blum. Peekaboom: A Game for Locating Objects in Images. In ACM SIGCHI Conference on Human Factors in Computing Systems, 2006.

[53] L. von Ahn, B. Maurer, C. McMillen, D. Abraham, and M. Blum. recaptcha: Human-based character recognition via web security measures Science, pages 1465-1468, 2008.

[54] I. Weber, S. Robertson, and M. Vojnovic. Rethinking the ESP Game. Technical report, Microsoft Research MSR-TR-2008-132, 2008.

[55] D. H. Wilson, A. C. Long, and C. Atkeson. A context-aware recognition survey for data collection using ubiquitous sensors in the home. In ACM SIGCHI Conference on Human Factors in Computing Systems, 2005.

[56] Y. Yang, B. B. Zhu, R. Guo, L. Yang, S. Li, and N. Yu. A comprehensive human computation framework: with application to image labeling. In A. El-Saddik, S. Vuong, C. Griwodz, A. D. Bimbo, K. S. Candan, and A. Jaimes, editors, ACM Multimedia, pages 479-488. ACM, 2008. 